\section{In the idiopathic inflammatory myopathies, reactive oxygen species are at the crossroad between immune and non-immune cell-mediated mechanisms}

\section{Dear editor,}

We read with great interest the article by Lightfoot et $a l^{1}$ who suggested that in the idiopathic inflammatory myopathies (IIM), reactive oxygen species (ROS) contribute to muscle weakness. The authors opposed immune and non-immune cell-mediated mechanisms in IIM and reviewed ROS as part of the latter. Here, we would like to point out recent data that reconcile this dichotomy and stress the interplay between ROS and immune cell-mediated processes during IIM.

In accordance with the authors' hypothesis, we recently observed high ROS formation, along with mitochondrial respiratory chain dysfunctions, in muscle of patients with dermatomyositis. $^{2}$ Furthermore, in muscle cells cultured in the absence of immune cells, interferon- $\beta$-induced mitochondrial dysfunctions in a ROS-dependent manner. Finally, in an antigen-induced mouse model of IIM, ROS scavenging with $\mathrm{N}$-acetyl cysteine prevented muscle weakness and mitochondrial dysfunctions and immunize cell infiltrate in muscle. ${ }^{2}$

Thus, ROS and immune cells are not independent actors in IIM. Indeed, ROS participate in immune cell infiltrate, which in turn can further increase ROS formation and mediated damages in the muscle. ${ }^{3}$ This definitely places ROS as central actors and potential therapeutic targets in IIM.
Alain Meyer, ${ }^{1,2}$ Jean Sibilia, ${ }^{2}$ Bernard Geny ${ }^{1}$

${ }^{1}$ Physiologie - Exploration fonctionnelle musculaire, EA 3072

${ }^{2}$ Centre de Référence des Maladies Auto-immune Rares, Hôpitaux Universitaires de Strasbourg, Strasbourg, France

Correspondence to Dr Alain Meyer, Exploration fonctionnelle musculaire, Hôpitaux Universitaires de Strasbourg de Strasbourg, Fédération de Médecine Translationnelle, Strasbourg 67000, France; alain.meyer1@chru-strasbourg.fr

Competing interests None declared.

Provenance and peer review Not commissioned; internally peer reviewed.

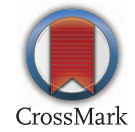

To cite Meyer A, Sibilia J, Geny B. Ann Rheum Dis 2015;74:e62.

Accepted 24 July 2015

Published Online First 14 August 2015

\section{Linked}

http://dx.doi.org/10.1136/annrheumdis-2015-208300

Ann Rheum Dis 2015;74:e62. doi:10.1136/annrheumdis-2015-208297

\section{REFERENCES}

1 Lightfoot AP, McArdle A, Jackson MJ, et al. In the idiopathic inflammatory myopathies (IIM), do reactive oxygen species (ROS) contribute to muscle weakness? Ann Rheum Dis 2015;74:1340-6.

2 Meyer A, Laverny G, Prevel N, et al. Interferon-beta induced reactive oxygen species participate in muscle inflammation and mitochondrial oxidative phosphorylation defects contributing to dermatomyositis muscle impairment. Ann Rheum Dis 2015;74(Suppl 2):119.

3 Mittal M, Siddiqui MR, Tran K, et al. Reactive oxygen species in inflammation and tissue injury. Antioxid Redox Signal 2014;20:1126-67. 